\title{
Quantum confinement corrections to the capacitance of gated one-dimensional nanostructures
}

\author{
K. M. Indlekofer* and J. Knoch \\ Institute of Thin Films and Interfaces (ISG-1) and Center of Nanoelectronic Systems for Information Technology (CNI), \\ Research Centre Jülich GmbH, D-52425 Jülich, Germany \\ J. Appenzeller \\ IBM T. J. Watson Research Center, P.O. Box 218, Yorktown Heights, New York 10598, USA
}

(Received 15 August 2006; published 27 September 2006)

\begin{abstract}
With the help of a multiconfigurational Green's function approach we simulate single-electron Coulomb charging effects in gated ultimately scaled nanostructures which are beyond the scope of a self-consistent mean-field description. From the simulated Coulomb-blockade characteristics we derive effective system capacitances and demonstrate how quantum confinement effects give rise to corrections. Such deviations are crucial for the interpretation of experimentally determined capacitances and the extraction of applicationrelevant system parameters.
\end{abstract}

DOI: 10.1103/PhysRevB.74.113310

PACS number(s): 73.63.-b, 72.10.-d, 73.22.-f, 73.23.-b

One of the major challenges for the simulation of electronic transport in low-dimensional nanostructures consists in an adequate description of the Coulomb interaction. In ultimately scaled semiconductor nanosystems, only a few electrons constitute the current. Thus, the details of the electron-electron interaction may become crucial for the electronic properties, as can be seen in experimentally observed single-electron charging effects in carbon nanotubes and III-V nanowhiskers. ${ }^{1,2}$ The channel region of a typical application-relevant nanostructure involves on the order of 100 single-particle states (or sites) and can be highly inhomogeneous and anisotropic. Here, external contacts and gate electrodes in general introduce nonlinear perturbations to the system. Hence, highly idealized interacting few-level models with a small number of effective parameters (constants) become inadequate. For a realistic simulation of quantum transport in application-relevant nanodevices, various approaches have been used, differing in the formulation of Coulomb interaction and contact coupling. While the orthodox theory ${ }^{3,4}$ correctly describes few-electron effects such as single-electron tunneling in quasi-isolated quantum dot systems, the nonequilibrium Green's function (NEGF) formalism $^{5-8}$ also accounts for contact renormalization and dissipation terms. However, the NEGF formalism becomes unable to describe the details of charging effects of a few fluctuating electrons as soon as a mean-field approximation is introduced.

Recently, we have presented a multiconfigurational selfconsistent Green's function (MCSCGF) approach, ${ }^{9}$ which represents a consistent extension of the mean-field NEGF method for the inclusion of single-electron effects under application-relevant conditions. This approach combines the simplicity and scalability of the mean-field NEGF approach with a many-body Fock space description of the Coulomb interaction of those electrons that are resonantly trapped within the nanostructure. As a key element, the algorithm identifies "relevant" trapped single-particle states that are subject to occupation fluctuations. Within the resulting relevant Fock subspace, many-body "configurations" $\kappa$ and associated weights $w_{\kappa}$ are defined as eigenstates and eigenvalues of the projected many-body statistical operator $\rho^{M B}$, respectively. Consequently, the system Green's functions are written as weighted averages $\bar{G}=\sum_{\kappa} w_{\kappa} G[\kappa]$, where $G[\kappa]$ follows from a Dyson equation with a suitable self-energy approximation $\Sigma[\kappa]$. As a self-consistency condition, $\rho^{M B}$ is obtained by use of the projected many-body Hamiltonian $H^{M B}$ and the subsidiary condition that $G^{M B}$ should resemble $\bar{G}$ within the relevant subspace, where $G^{M B}$ is calculated via $\rho^{M B}$ and $H^{M B}$.

In this Brief Report we show how the MCSCGF formalism can be used to calculate electronic transport properties of gated one-dimensional (1D) nanosystems in the singleelectron tunneling regime beyond the mean-field approach. Furthermore, we extract realistic system capacitances from the simulated $I-V$ characteristics. Three length scales are relevant for the Coulomb interaction energies of the system: the geometrical length $L$, the electrostatic screening length $\lambda$, and the de Broglie wavelength of the electron. The latter becomes important in a nanoscale system with quantum confinement, giving rise to significant deviations from the expected capacitances.

In the following, we discuss a 1D nanostructure (i.e., one lateral mode only) as depicted in Fig. 1. In the shown coaxial geometry, the channel is surrounded by a metallic gate electrode, separated by an insulating oxide layer. The 1D channel of the nanostructure is connected to two Schottky contacts (denoted as source and drain) which serve as electron reservoirs. ${ }^{14}$ In order to visualize the electronic structure of the system, a radial cut through the inner region of the structure has been inserted, showing the ground state probability density $|\psi(x)|^{2}$ and the associated local density of states (LDOS) for one electron inside the channel. For the discussion of interaction effects we will now consider an example of an ultimately scaled semiconducting nanocolumn (see also Ref. 9) with an effective channel length of $L$ $=19 \mathrm{~nm}$, a channel diameter of $d_{c h}=4 \mathrm{~nm}$, and an oxide thickness of $d_{o x}=10 \mathrm{~nm}$. Furthermore, we chose $\epsilon_{c h}=15$ and $\epsilon_{o x}=3.9$, yielding a screening length of $\lambda \approx 3.7 \mathrm{~nm}$. The latter is obtained from 


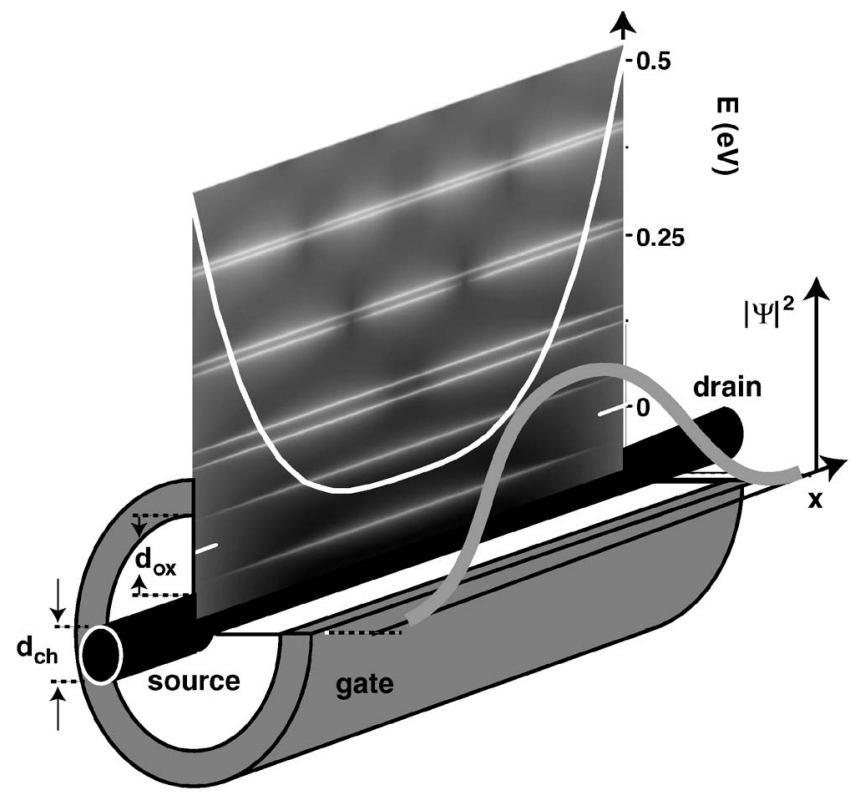

FIG. 1. Schematic sketch of the considered coaxially gated nanosystem (see text for more details).

$$
\lambda^{2}=\frac{\epsilon_{c h}}{\epsilon_{o x}} \frac{d_{c h}^{2}}{8} \ln \left(1+2 \frac{d_{o x}}{d_{c h}}\right)
$$

for a coaxial system layout. ${ }^{10}$ The Schottky source and drain contacts exhibit a barrier of $\Phi_{B}=0.5 \mathrm{eV}$ (with respect to the Fermi energy), whereas the effective electron mass is taken as $m^{*}=0.05 m_{e}$. This parameter set was chosen in order to clearly visualize the Coulomb effects, at the same time being close enough to experimental realizations (e.g., InGaAsbased whiskers with $\mathrm{SiO}_{2}$ coating).

Figure 2(a) shows a simulated gray scale map of the drain current as a function of the applied gate and drain voltages in the single-electron tunneling regime $(T=77 \mathrm{~K}) .{ }^{15}$ One can clearly identify the typical diamondlike shaped black areas of Coulomb blockade with a suppressed drain current. Darkgray areas correspond to electronic transport through the lowest longitudinal resonantly trapped state, whereas brighter areas involve the first (and higher) excited resonances. Such a behavior is beyond the capabilities of a meanfield NEGF approach that is based on a self-consistent potential, demonstrating the strengths of the employed multiconfigurational approach. In order to visualize the interaction mechanism that goes beyond the mean field, Fig. 2(b) shows the local density of states within the channel region at different voltage conditions and the associated mean-field potentials. For the empty channel " 0 ," we obtain a set of quasibound states and resulting resonances. With one electron inside the channel near equilibrium point " 1 ," one electron occupies the lowest state and all further states are shifted to higher energies by the Coulomb interaction. Note that the electron does not experience its own Hartree potential. Finally, under strong nonequilibrium bias conditions at point " $X$," the spectral properties result from a weighted mixture of an empty channel and a channel containing one electron.

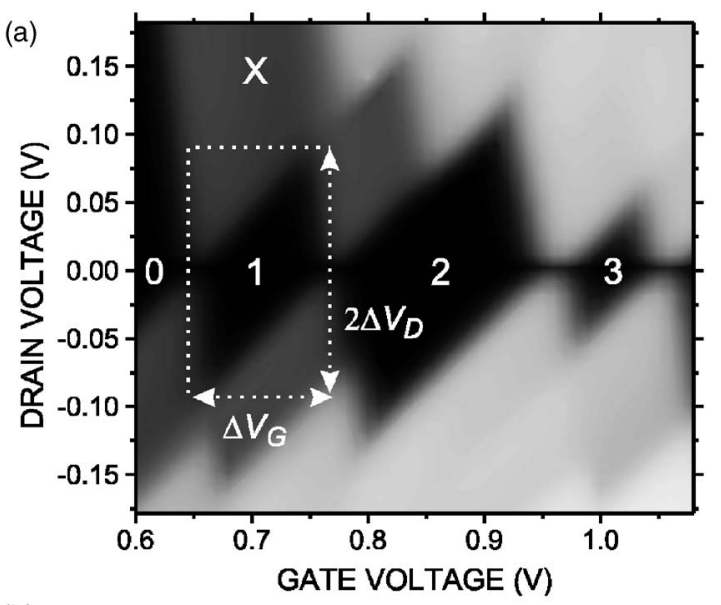

(b)
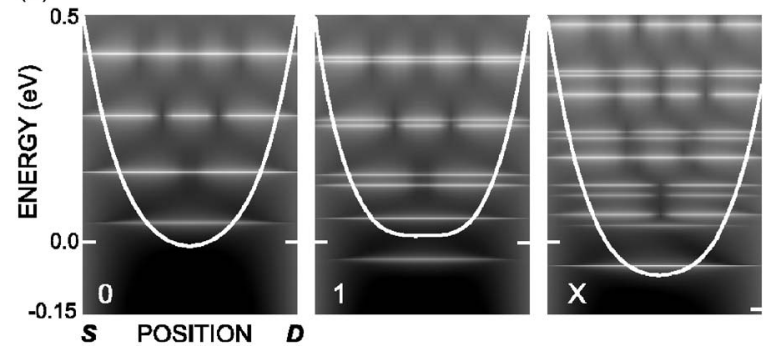

FIG. 2. (a) Simulated single-electron tunneling characteristics as a function of the applied gate and drain voltages for $T=77 \mathrm{~K}$. Here, the absolute value of the drain current is mapped to a nonlinear gray scale. Within the black diamonds of Coulomb blockade the electron number becomes quantized as indicated by white numbers. $\Delta V_{G}$ and $\Delta V_{D}$ denote the width and half-height of the first diamond, respectively. (b) Corresponding local density of states and meanfield potential within the channel for selected points $0,1, X$ in map (a). Point $X$ corresponds to a nonequilibrium state with electron number $\approx 0.3$.

Here, the electronic properties clearly deviate from a meanfield picture.

For the considered example, the height of the Coulomb diamond with one electron is determined by the Coulomb interaction of two electrons with opposite spin within the lowest channel resonance (ground state). In contrast, for the second diamond one has to consider not only the interaction between the lowest and the first excited states but also the difference between the single-particle energies of the two involved states. Hence, its size is significantly enlarged. These observations strongly depend on the degeneracy of the considered channel resonances and on the ratio of quantization and Coulomb energies. In ultimately scaled systems that are addressed in this paper, the longitudinal quantization energy dominates and it is justified to consider the ground state Coulomb matrix element only. In contrast, for longer channels, the quantization energy may become much smaller than the charging energy and we could end up with a situation where the occupation of higher excited states (with a reduced Coulomb matrix element) and a reorganization of electrons might become energetically favorable for the addition of the next electron ${ }^{11}$ (and Wigner molecules might form ${ }^{12}$ ). In the following, we restrict ourselves to the discussion of the low- 
est Coulomb diamond which corresponds to the onset of the current flow.

In general, effective quantities such as the gate capacitance $C_{G}^{*}$ and the total system capacitance $C_{\Sigma}^{*}$ can be extracted from the shape of the observed Coulomb diamonds ${ }^{13}$ (provided that the latter is solely due to Coulomb interaction): From the total width $\Delta V_{G}$ and half-height $\Delta V_{D}$ one obtains $C_{G}^{*}=e / \Delta V_{G}$ and $C_{\Sigma}^{*}=e / \Delta V_{D}$, respectively. In our example (Fig. 2), we have $\Delta V_{G} \approx 114 \mathrm{mV}$ and $2 \Delta V_{D}$ $\approx 186 \mathrm{mV}$, and therefore $C_{G}^{*} \approx 1.41 \mathrm{aF}$ and $C_{\Sigma}^{*} \approx 1.72 \mathrm{aF}$. Consequently, a Coulomb interaction energy of $E_{\Sigma}^{*}=e^{2} / C_{\Sigma}^{*}$ $\approx 93 \mathrm{meV}$ can be derived (which is identical to a twoparticle Coulomb matrix element; see Ref. 9). Note that, for such ultrashort nanosystems, where the quantization effects play an important role, these capacitances in general are voltage-dependent quantities, depending on the wave functions of the involved longitudinal channel resonances that are being filled at the considered electrode voltages.

For the classical capacitance of the considered coaxial geometry of the $1 \mathrm{D}$ channel region one obtains

$$
C_{o x}=\frac{\pi \epsilon_{0} \epsilon_{c h} d_{c h}^{2} L}{4 \lambda^{2}}=2 \pi \epsilon_{0} \epsilon_{o x} \frac{L}{\ln \left(1+2 d_{o x} / d_{c h}\right)} .
$$

Employing the Coulomb Green's function (for details see Ref. 9) for fixed potential boundary conditions, the Coulomb interaction energy $E_{\Sigma}^{\text {hom }}$ of a homogeneously distributed test charge $e$ with an identical background charge thus reads as $E_{\Sigma}^{\text {hom }}=e^{2} / C_{\Sigma}^{\text {hom }}$ with the total homogenous system capacitance

$$
C_{\Sigma}^{h o m}=C_{o x}\left(1-2 \frac{\cosh (L / \lambda)-1}{(L / \lambda) \sinh (L / \lambda)}\right)^{-1} .
$$

(Note that $C_{\Sigma}^{\text {hom }}>C_{o x}$ and $C_{\Sigma}^{\text {hom }} \rightarrow C_{o x}$ for $L / \lambda \rightarrow \infty$.) Using the given parameters, we thus obtain $C_{o x}=2.30 \mathrm{aF}$ and $C_{\Sigma}^{\text {hom }}=3.75 \mathrm{aF}$. In this context, we therefore define the quantity

$$
f_{\Sigma}=\frac{e^{2}}{C_{\Sigma}^{*}} / \frac{e^{2}}{C_{\Sigma}^{\text {hom }}}=C_{\Sigma}^{\text {hom }} \Delta V_{D} / e,
$$

which we refer to as the "quantum confinement factor" for the given geometrical structure and parameters (voltages, etc.). In the example above, we have $f_{\Sigma} \approx 2.2$. The observation that $f_{\Sigma}$ becomes greater than 1 for ultimately scaled structures can easily be understood if one takes a look at the quasibound single-electron wave function (Fig. 1) of the considered Coulomb diamond: Typically, this wave function exhibits an enhanced probability amplitude in the center region of the 1D channel due to quantum confinement in the transport direction which is a result of the Schottky barriers at the source and drain contacts. In other words, the electron is spatially confined within a reduced effective channel length below $L$. This implies an increased Coulomb interaction energy $E_{\Sigma}^{*}=93 \mathrm{meV}$ compared to $E_{\Sigma}^{\text {hom }}=43 \mathrm{meV}$ for a homogeneous charge distribution, or equivalently $C_{\Sigma}^{*}<C_{\Sigma}^{\text {hom }} \cdot{ }^{16}$ Consequently, larger voltage changes are required to add one further electron to the channel region. This implies that care has to be taken with the extraction of geometrical parameters
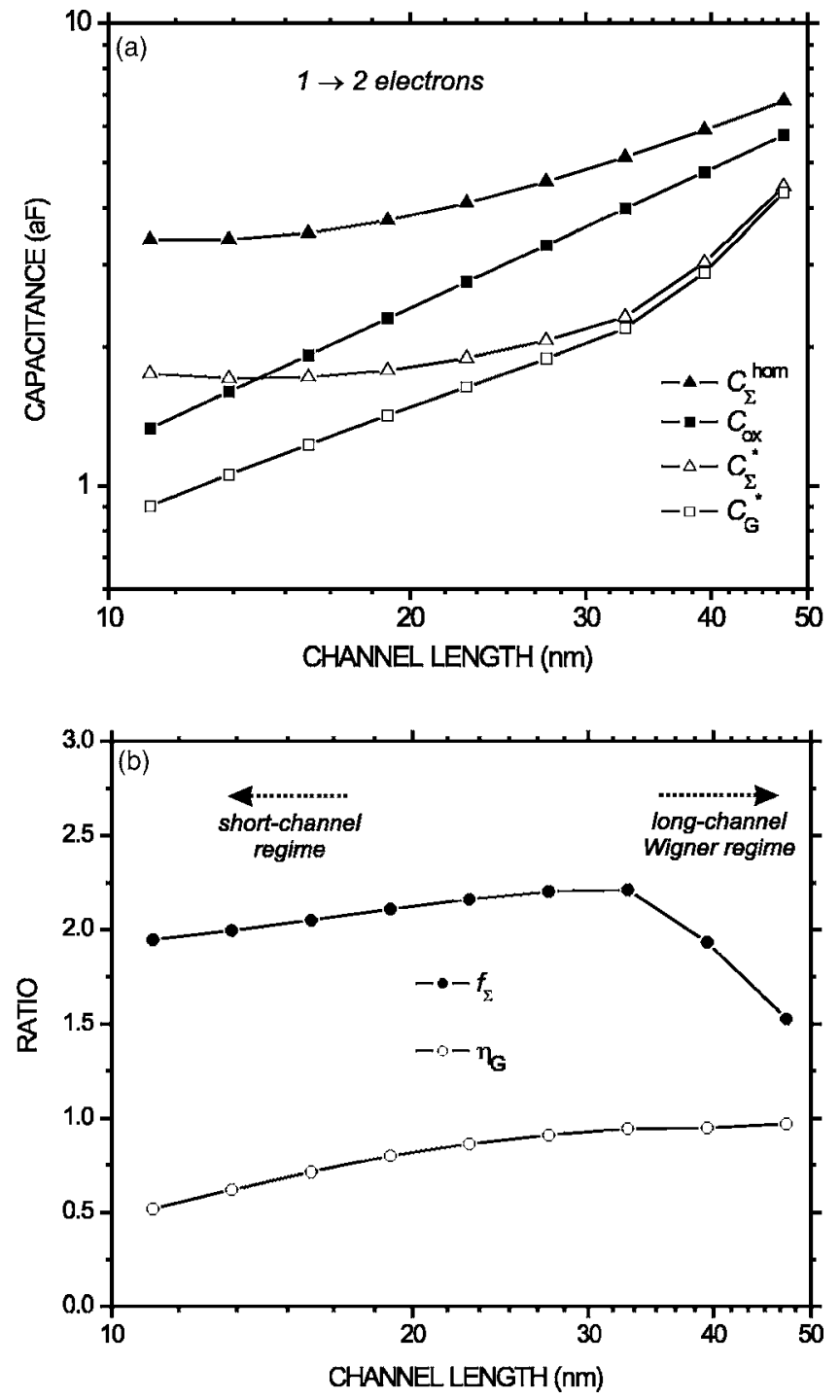

FIG. 3. (a) Simulated capacitances for the first two electrons as a function of the channel length $L$ with an adaptive gate voltage. The screening length $\lambda \approx 3.7 \mathrm{~nm}$ and the effective mass $m^{*}$ $=0.05 m_{e}$ are kept constant. (b) Quantum confinement factor $f_{\Sigma}$ $=C_{\Sigma}^{\text {hom }} / C_{\Sigma}^{*}$ and gate efficiency $\eta_{G}=C_{G}^{*} / C_{\Sigma}^{*}$ as derived from (a).

from experimental data as will be discussed in more detail below.

Figure 3(a) shows the simulated capacitances for the first two electrons (ground state wave function and next higher state) as a function of the channel length $L$ for a fixed $\lambda$ and $m^{*}$. Here, the gate voltage $V_{G}$ is chosen adaptively such that the ground state energy coincides with the Fermi energy of the source contact (for a constant Schottky barrier height $\Phi_{B}=0.5 \mathrm{eV}$ with $\left.V_{D} \equiv V_{S} \equiv 0\right)$. For each $L$, the wave function and the associated Coulomb interaction energy $E_{\Sigma}^{*}=e^{2} / C_{\Sigma}^{*}$ have been obtained via numerical diagonalization of the channel Hamiltonian. ${ }^{9}$ As expected, for larger $L / \lambda$ we observe that $C_{\Sigma}^{*} \rightarrow C_{G}^{*}$ and $C_{\Sigma}^{\text {hom }} \rightarrow C_{o x}$, which means that the source and drain influence becomes negligible in this limit. On the other hand, for the ultrashort structure with $L$ $<20 \mathrm{~nm}$, one can clearly identify the increasing influence of the source and drain capacitances. Therefore, an increased 
gate voltage becomes necessary to pull the lowest quasibound state down to the Fermi energy of the source contact for a fixed Schottky barrier height (e.g., $V_{G}=0.52 \mathrm{~V}$ for $L$ $=50 \mathrm{~nm}$, and $V_{G}=1.1 \mathrm{~V}$ for $L=10 \mathrm{~nm}$ ). This is described quantitatively by the efficiency $\eta_{G}=C_{G}^{*} / C_{\Sigma}^{*}=\Delta V_{D} / \Delta V_{G}$ of the gate electrode at changing the considered quasibound energy level, as plotted in Fig. 3(b).

Most noticeably for all ranges of $L / \lambda$, there is a significant deviation of $C_{\Sigma}^{*}$ from $C_{\Sigma}^{\text {hom }}$ (and $C_{G}^{*}$ from $C_{o x}$ ) as can be seen in Fig. 3(a). This observation, in fact, is described by the quantum confinement factor $f_{\Sigma}$ as plotted in Fig. 3(b). In the short-channel regime, we obtain $f_{\Sigma}>1$, which can be explained by the longitudinal confinement of the ground state wave function and the resulting increased Coulomb interaction. Furthermore, as can be seen in Fig. 3, the onset of the short-channel regime, where $\eta_{G}$ deviates significantly from 1 due to increasing source and drain influence, is shifted to smaller channel lengths $L<30 \mathrm{~nm}$ compared to $L>50 \mathrm{~nm}$ where $C_{\Sigma}^{\text {hom }}$ starts to deviate from $C_{o x}$. Again, this finding can be attributed to the longitudinal confinement of the wave function with a reduced electron charge density at the source and drain contacts. For larger channel lengths $L$, however, we observe a rapid decrease of $f_{\Sigma}$ (or equivalently, a strong increase of $C_{\Sigma}^{*}$ and $C_{G}^{*}$ ). This finding can be attributed to the onset of a Wigner regime due to a significantly reduced average electron density (because of an increasing spatial separation of the two considered electrons compared to $\lambda)$.

The discussed confinement effect becomes very important for the experimental determination of application-relevant capacitances from measured Coulomb diamonds. Analogous to the interpretation of our simulation results above, effective capacitances $C_{\Sigma}^{*}$ and $C_{G}^{*}$ can be calculated from the experimentally determined values of $\Delta V_{D}$ and $\Delta V_{G}$ at low temperatures (typically $4 k_{B} T<E_{\Sigma}^{*}$ ). One has to be careful with the interpretation of these effective capacitances as far as the derivation of geometrical parameters from the latter and their relevance at room temperature are concerned. For example, it is possible to calculate $d_{o x}$ from $C_{\Sigma}^{*}$. By inspecting Eqs. (1)-(4), however, one can see that this is a nontrivial task since $\lambda$ depends on $d_{o x}$, and $f_{\Sigma}$ (which results from a Coulomb matrix element) in turn depends on $\lambda$. If we identified $C_{\Sigma}^{\text {hom }}$ with $C_{\Sigma}^{*} \equiv e / \Delta V_{D}$ (i.e., setting $f_{\Sigma} \equiv 1$ ) from experimental data, we would overestimate $d_{o x}$. In the considered example, we would end up with $d_{o x}>50 \mathrm{~nm}$ (instead of $10 \mathrm{~nm}$ ) which is a consequence of the logarithm in Eqs. (1) and (2).

In conclusion, we have demonstrated that the multiconfigurational self-consistent Green's function approach is able to model single-electron Coulomb charging effects in ultimately scaled gated 1D nanostructures, providing a means for the determination of interaction energies and resulting effective capacitances. In turn, a quantitative discussion of quantum confinement corrections to the system capacitance was given, resulting in a significant deviation from the classical capacitance, which has an immediate consequence for the interpretation of experimental system parameters.
*Electronic address: m.indlekofer@fz-juelich.de

${ }^{1}$ M. Suzuki, K. Ishibashi, K. Toratani, D. Tsuya, and Y. Aoyagi, Appl. Phys. Lett. 81, 2273 (2002).

${ }^{2}$ C. Thelander, T. Martensson, M. T. Bjoerk, B. J. Ohlson, M. W. Larsson, L. R. Wallenberg, and L. Samuelson, Appl. Phys. Lett. 83, 2052 (2003).

${ }^{3}$ C. W. J. Beenakker, Phys. Rev. B 44, 1646 (1991).

${ }^{4}$ D. V. Averin, A. N. Korotkov, and K. K. Likharev, Phys. Rev. B 44, 6199 (1991).

${ }^{5}$ R. Lake, G. Klimeck, R. C. Bowen, and D. Jovanovic, J. Appl. Phys. 81, 7845 (1997).

${ }^{6}$ S. Datta, Electronic Transport in Mesoscopic Systems (Cambridge University Press, Cambridge, UK, 1995).

${ }^{7}$ R. Venugopal, Z. Ren, S. Datta, M. S. Lundstrom, and D. Jovanovic, J. Appl. Phys. 92, 3730 (2002).

${ }^{8}$ K. Alam and R. Lake, Appl. Phys. Lett. 87, 073104 (2005).

${ }^{9}$ K. M. Indlekofer, J. Knoch, and J. Appenzeller, Phys. Rev. B 72, 125308 (2005).
${ }^{10}$ C. P. Auth and J. D. Plummer, IEEE Electron Device Lett. 18, 74 (1997).

${ }^{11}$ K. M. Indlekofer and H. Lüth, Phys. Rev. B 62, 13016 (2000).

${ }^{12}$ P. Jarillo-Herrero, S. Sapmaz, C. Dekker, L. P. Kouwenhoven, and H. S. J. van der Zant, Nature (London) 429, 389 (2004).

${ }^{13}$ H. Grabert and M. H. Devoret, Single Charge Tunneling (Plenum Press, New York, 1992).

${ }^{14}$ The considerations remain valid qualitatively for general contact barriers.

${ }^{15}$ In this example, up to 64 configurations of relevant states with a diagonal interaction approximation were considered.

${ }^{16}$ The reduction of $C_{\Sigma}^{*}$ with respect to $C_{\Sigma}^{\text {hom }}$ is not to be confused with the concept of a finite quantum capacitance $C_{Q}=e^{2} \bar{D}\left(E_{F}\right)$ with a thermally averaged density of states $\bar{D}\left(E_{F}\right)$ [S. Luryi, Appl. Phys. Lett. 52, 501 (1987)]. The considered nanosystem in the single-electron regime exhibits a quasi-zero-dimensional electronic structure, where $C_{Q}$ equals 0 or $\infty$. 